\title{
Ir caminando al trabajo previene la hipertensión arterial.
}

Walking to work and the risk for hypertension in men: The Osaka Health Survey. Hayashi T, Tsumura K, Suematsu C et al. Annals of Internal Medicine. 1999;131:21-26.

\section{Objetivo}

Investigar la asociación entre la duración de la caminata al trabajo y la actividad física en el tiempo libre con el riego de hipertensión arterial (HTA).

\section{Diseño}

Estudio prospectivo de cohortes*.

\section{Lugar}

Osaka, Japón.

\section{Participantes}

La cohorte incluyó 6017 hombres japoneses de 35 a 60 años de edad, cuyo trabajo era considerado como sedentario. Debían tener al inicio del seguimienro la tensión arterial sistólica (TAS) $<140$ $\mathrm{mmHg}$, la tensión arterial diastólica $(T A D)<90 \mathrm{mmHg}$, una curva de tolerancia oral a la glucosa normal y no tener historia de HTA o diabetes previa.

\section{Evaluación de factores de riesgo}

Los datos de la actividad física fueron obtenidos usando cuestionarios, donde se incluían preguntas sobre comportamientos relacionados con la salud como fumar, consumo diario de alcohol, etc. La actividad física fue medida como duración de la caminata al trabajo: $<10$ minutos, 11-20 minutos $y>20$ minutos.

También fue evaluada la actividad física en el tiempo libre (fuera del trabajo) siempre que realizaran ejercicio físico regular (jogging, ciclismo, natación, tenis, o que dure 30 minutos o mas). Se la clasificó en: < a 1 vez por semana, 1 vez por semana, > 2 veces por semana.

\section{Medición de resultados principales}

La medición de la presión arterial fue hecha con técnicas estándares por enfermeras profesionales. Se utilizó un valor de por lo menos $160 / 95$ mm Hg como criterio diagnóstico de HTA. También se registró el índice de masa corporal (IMC).

\section{Resultados principales}

Durante 59784 años/persona de seguimiento fueron confirmados 626 nuevos casos de HTA. La duración de la caminata al trabajo estuvo asociada con una disminución en el riesgo de desarrollar HTA. El riesgo relativo (RR) ajustado (por análisis multivariado*) fue de 1 para caminatas < 10 minutos (categoría de referencia), 0,88 (IC95\% 0,75-1,04) para caminatas entre 11 a 20 minutos y 0,71 $($ IC95\% 0,52-0,97) para caminatas $>20$ minutos ( $p$ de tendencia $=$ $0,02)$.

El RR ajustado para HTA disminuyó de 1 para hombres que realizaban actividad física en el tiempo libre menos de una vez por semana (categoría de referencia) a 0,62 para aquellos que realizaban ejercicios 1 vez por semana (IC95\% 0,45-0,85) y a 0,74 para $>2$ veces por semana (IC95\% 0,60-0,90). Por cada 26,3 hombres que caminen al trabajo, podría prevenirse 1 caso de HTA $(N N T=26,3)$.

\section{Conclusiones}

Caminar al trabajo y otros tipos de actividad física disminuyen el riesgo para HTA en hombres japoneses. El ejercicio regular puede prevenir la HTA.

\section{COMENTARIO}

Los primeros estudios epidemiológicos realizados para analizar la relación entre sedentarismo y enfermedades crónicas fueron de tipo retrospectivo. ${ }^{1-4}$ Analizaron grandes poblaciones incluyendo datos de ciudades 0 estados enteros, en base fundamentalmente, a las causas de muerte especificadas en los certificados de defunción y al tipo de ocupación laboral consignados. Los estudios prospectivos presentan datos muchos mas precisos y confiables en cuanto al mayor o menor gasto energético de las actividades laborales y a las causas de morbimortalidad. Además la mayoría de ellos incluyen en el análisis la actividad física extralaboral. Los datos prospectivos de este estudio muestran que la duración de la caminata al trabajo estaba asociada con un riesgo disminuido en la incidencia de HTA; aún luego del ajuste por edad, IMC, consumo de alcohol, actividad física en el tiempo libre, tabaquismo, TAS y TAD basales y glucemia en ayunas. El análisis multivariado también demostró que el ejercicio físico regular como mínimo de una vez por semana estaba inversamente relacionado con el riesgo de HTA. La homogeneidad relativa de la cohorte aumenta en cierta forma la validez interna* del estudio; debido a la relativa uni- formidad en los antecedentes educativos y socioeconómicos de la muestra. El estudio tiene algunas limitaciones potenciales en especial en cuanto a la validez externa*. Primero, todos los participantes eran empleados de la misma compañía, pudiendo los resultados no ser representativos de la población general, pero pareciera que la asociación encontrada podría ser aplicada a hombres que trabajan fuera de su hogar. Segundo, todos los participantes de este estudio eran hombres; aun falta determinar si los resultados pueden ser aplicados a mujeres; y tercero, podría suceder que la población japonesa se comporte de manera diferente (genética, hábitos dietarios, factores desconocidos). Sin embargo los resultados proveen suficiente evidencia de que la duración de la caminata al trabajo tiene un efecto independiente sobre el riesgo de HTA. Aún las personas que manejan al trabajo o que usan transporte público podrían beneficiarse estacionando o dejando su transporte a más de 20 minutos de caminata al trabajo. Además de la recomendación de caminar al trabajo los médicos no debemos olvidar el control del peso, el consumo adecuado de alcohol y el aumento de la actividad física en el tiempo libre.

*Ver glosario

Dr. Christian Rhaiel

Unidad de Medicina Familiar y Preventiva. Hospital Italiano de Buenos Aires.

Referencias

1. Leon AS. Physical activity levels and coronary heart disease. Analysis of epidemiologic and supporting studies. Med Clin North Am. 1985;65:3-20.

2. Paffenbarger RS Jr, Jung DL, Leung RW, Hyde RT. Physical activity and hypertension: an epidemiological view. Ann Med. 1991; 23:319-27.

3. Kingwell BA, Jenninngs GL. Effect of walking and other exercise programs upon blood pressure in normal subjets. Med J Aust. 1993; 158:234-8.

4. Appel LJ, Moore TJ, Obarzanek E, Vollmer WM, Svetkey LP, Sacks FM et al. A clinical trial of the effects of dietary patterns on blood pressure. DASH Collaborative Research Group. N Engl J Med. 1997; 336: 1117-24 